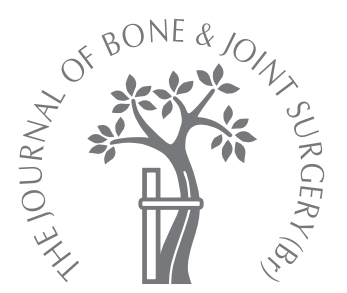

S. S. Rajaratnam, C. Jack,

A. Tavakkolizadeh,

M. D. George,

R. J. Fletcher,

M. Hankins,

J. A. N. Shepperd

From Conquest

Hospital, St

Leonards-on-Sea,

England

I. S. Rajaratnam, FRCS (Tr \& Orth), BSc (Hons), Senior Hip \& Knee Fellow

Peninsula Orthopaedic

Research Institute, 812

Pittwater Road, Deewhy,

Sydney, Australia.

C. Jack, MRCS, Specialist Registrar

Queen Elizabeth Hospital,

Woolwich, London SE18 4QH, UK.

- A. Tavakkolizadeh, FRCS (Tr \& Ortho), MSc, Specialist Registrar

Kings College Hospital, Denmark Hill, London SE5 9RS, UK.

- M. D. George, FRCS (Tr \& Orth), Hip Fellow

Guy's Hospital, St Thomas' Street London Bridge, SE1 9RT, UK

R. J. Fletcher, MRCS, BSc(Hons), Research Registrar I. A. N. Shepperd, FRCS, Consultant Orthopaedic Surgeon Conquest Hospital, The Ridge, St Leonards-on-Sea, East Sussex TN37 7RD, UK

M. Hankins, BSc(Hons), FRSS, Senior Research Fellow/ Statistician

Brighton \& Sussex Medical School, Falmer BN1 9RH, UK.

Correspondence should be sent to $\mathrm{Mr} \mathrm{S.} \mathrm{S.} \mathrm{Rajaratnam;} \mathrm{e-mail:}$ srajaratnam@talk21.com

(C2008 British Editorial Society of Bone and Joint Surgery doi:10.1302/0301-620X.90B1. $19731 \$ 2.00$

$J$ Bone Joint Surg $[\mathrm{Br}]$ 2008;90-B:27-30.

Received 22 May 2007;

Accepted after revision 29

August 2007

\section{Long-term results of a hydroxyapatite-coated femoral component in total hip replacement}

\author{
A 15- TO 21-YEAR FOLLOW-UP STUDY
}

\begin{abstract}
Between 1986 and 1991 we implanted 331 consecutive Furlong hydroxyapatite-coated femoral components of a total hip replacement in 291 patients. A cemented acetabular prosthesis was used in 217 hips and a hydroxyapatite-coated component in 114. We describe the long-term clinical and radiological survival of the femoral component at a mean follow-up of 17.5 years (15 to 21$)$. Only two patients $(0.68 \%$ ) were lost to follow-up. With revision of the femoral component for any reason as the endpoint, the survival at a mean of 17 years was $97.4 \%$ (95\% confidence interval 94.1 to 99.5$)$, and with revision for aseptic loosening as the endpoint it was $100 \%$. The survival at a maximum of 21 years with revision of the femoral component for any reason as the endpoint was $97.4 \%$ (95\% confidence interval 81.0 or 99.5 ). These results compare favourably with the best long-term results of cemented or uncemented femoral components used in total hip replacement.
\end{abstract}

The results of the titanium hydroxyapatite (HA)-coated Furlong hip replacement (Joint Replacement Instrumentation Ltd, London, United Kingdom) have previously been reported, showing survival of the femoral component of $100 \%$ at ten years ${ }^{1}$ and of $99 \%$ at a mean of 13 years. ${ }^{2}$ We report the longest prospective series to date of an HA-coated femoral prosthesis.

\section{Patients and Methods}

Between 1986 and 1991, in Hastings, United Kingdom, we performed 331 total hip replacements (THRs) in 291 patients with a mean age of 71.2 years (31.1 to 89.8). A total of 40 patients had bilateral THRs, 30 under one anaesthetic and ten in a staged procedure. All the patients had a Furlong HA-coated THR. The operation was performed via a Watson-Jones approach by, or under the supervision of a consultant orthopaedic surgeon (JANS).

A total of 217 THRs were undertaken using a Furlong HA-coated femoral component and a Furlong ultra-high-density-polyethylene cemented acetabular component. The remaining 114 THRs, operated on after 1 January 1990, had an HA-coated threaded acetabular component (Joint Replacement Instrumentation Ltd) and a HA-coated Furlong femoral component. A $32 \mathrm{~mm}$ modular ceramic head was used in all patients. Each patient had three doses of prophylactic antibiotics (cefuroxime) and were allowed to bear weight fully immediately after surgery.

The patients were reviewed clinically and radiologically at $6,12,26$ and 52 weeks after operation and annually thereafter. Anteroposterior radiographs of the pelvis and lateral radiographs of the hips were taken and the Merle d'Aubigne and Postel hip score ${ }^{3}$ was used for the assessment of pain, mobility and function.

The stability and fixation of the femoral component was assessed by two independent observers (SSR, CJ) by a consensus of opinion. The appearance of radiolucencies around the component according to Gruen, McNiece and Amstutz ${ }^{4}$ was noted, as was subsidence on serial radiographs, the presence of increased bone density suggesting bony ingrowth, and the appearance of radiolucent lines and pedestal formation at the tip of the stem. An assessment of the radiographs was also made for evidence of stress shielding and of each Gruen zone for osteopenia.

\section{Results}

The patients were followed up for a mean of 17.5 years (15 to 21$)$. A total of 184 patients $(63.2 \% ; 211$ hips) had died by the final followup. All had been reviewed within one year of their death and were therefore included in the survival analysis.

Of the remaining 107 patients with 120 THRs, 15 failed to attend the final review and 


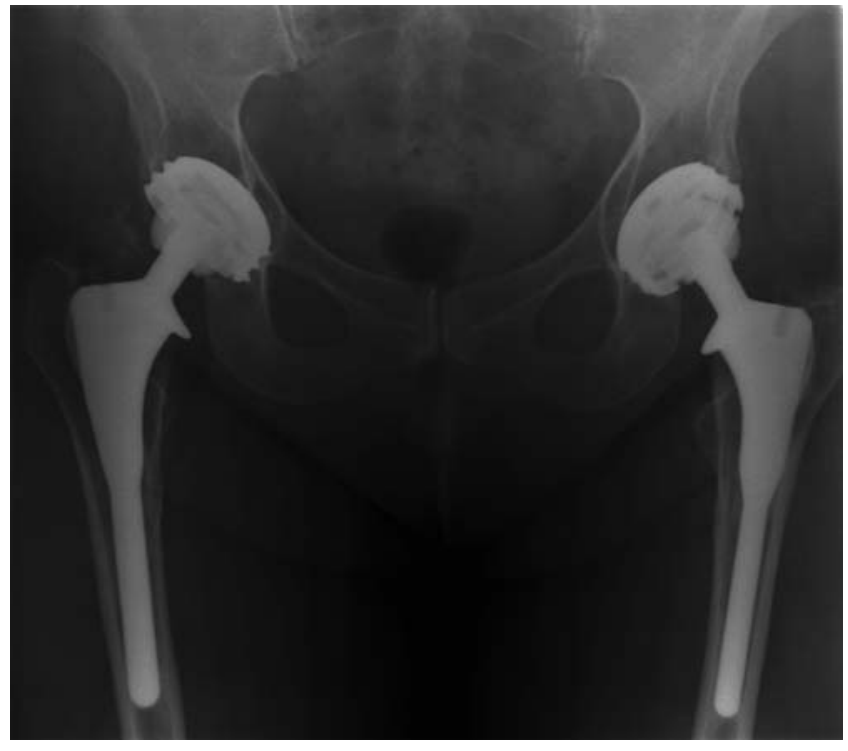

Fig. 1

Anteroposterior pelvic radiograph showing well-fixed bilateral Furlong hydroxyapatite-coated femoral components and threaded acetabular components, 17 years after total hip replacement.

were contacted via an extended telephone interview. Their results are included in the study. Two hips in two patients $(0.68 \%)$ were lost to follow-up. One had moved abroad 2.5 years after THR and could not be contacted. The other had a well functioning THR at six years post-operatively and refused further review.

Clinical and radiological. The mean Merle D'Aubigne and Postel $^{3}$ score recorded for the 92 patients (105 hips) who attended the latest follow-up was 5.63 (3 to 6) for pain, 5.42 (2 to 6 ) for mobility and 4.50 (0 to 6) for function.

No patient reported anterior thigh pain at any review.

Slight rounding of the femoral calcar under the collar was seen in 43 of 105 hips (41\%).

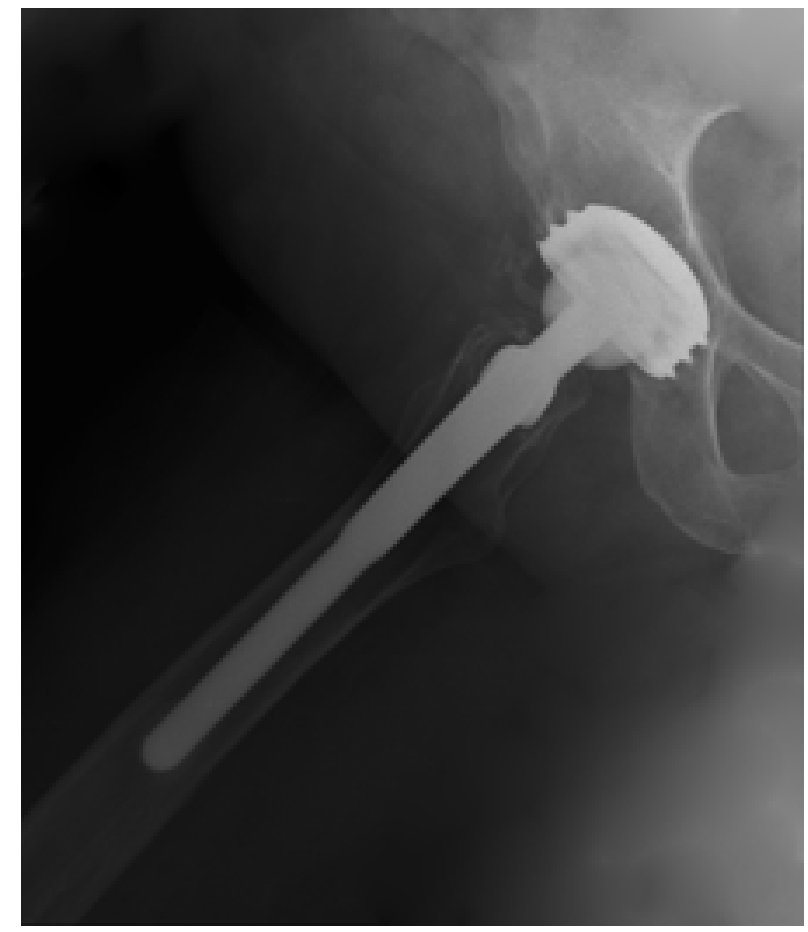

Fig. 2

Lateral radiograph of the hip showing a well-fixed Furlong hydroxyapatite-coated total hip replacement 17 years after operation.

There were no cases of aseptic loosening of the femoral component during the study period. In all cases, the femoral component remained well-fixed with no measured migration at the latest follow-up, with radiological evidence of bonding in the form of spot-weld formation ${ }^{2}$ into the stem and a blastic reaction at its tip (Figs 1 and 2). Six patients $(1.8 \%)$ underwent revision of the femoral component for trauma, sepsis or trunion fretting, at which stage their well-fixed femoral component was removed (Table I).

Table I. Details of the revisions of the femoral components

\begin{tabular}{|c|c|c|c|}
\hline Case & Time since THR $^{*}$ (yrs) & Indication for revision & Treatment \\
\hline 1 & 10 & $\begin{array}{l}\text { Traumatic loosening of both components following road } \\
\text { traffic accident }\end{array}$ & One-stage revision of femoral component \\
\hline 2 & 7 & Late infection following road traffic accident & Two-stage revision of both components \\
\hline \multirow[t]{2}{*}{3} & 13 & Loosening of the acetabular component & One-stage revision \\
\hline & & Infection & Two-stage revision of both components \\
\hline 4 & 1 & $\begin{array}{l}\text { Acetabular loosening leading to dissociation of ceramic head } \\
\text { from trunnion }\end{array}$ & $\begin{array}{l}\text { One-stage revision of femoral component } \\
\text { and acetabulum }\end{array}$ \\
\hline 5 & 9 & Sepsis & Two-stage revision of femoral component \\
\hline 6 & 8 & Peri-prosthetic fracture and loosening & One-stage revision of femoral component \\
\hline
\end{tabular}

* THR, total hip replacement 
Table II. Cumulative survival of the 331 hips (including the six revisions)

\begin{tabular}{|c|c|c|c|c|c|c|}
\hline Years since operation & Number of hips & Failures & Withdrawn & Number at risk & Cumulative survival (\%) & $95 \% \mathrm{Cl}^{*}$ \\
\hline 1 & 331 & 1 & 14 & 324.0 & 99.7 & 99.1 to 100.0 \\
\hline 2 & 316 & 0 & 7 & 312.5 & 99.7 & 99.1 to 100.0 \\
\hline 3 & 309 & 0 & 13 & 302.5 & 99.7 & 99.1 to 100.0 \\
\hline 4 & 296 & 0 & 22 & 285.0 & 99.7 & 99.1 to 100.0 \\
\hline 5 & 274 & 0 & 12 & 268.0 & 99.7 & 99.1 to 100.0 \\
\hline 6 & 262 & 0 & 15 & 254.5 & 99.7 & 99.0 to 100.0 \\
\hline 9 & 220 & 1 & 8 & 216.0 & 98.4 & 96.8 to 100.0 \\
\hline 10 & 211 & 1 & 12 & 205.0 & 98.0 & 96.1 to 99.8 \\
\hline 11 & 198 & 0 & 13 & 191.5 & 98.0 & 96.0 to 99.8 \\
\hline 12 & 185 & 0 & 25 & 172.5 & 98.0 & 96.0 to 99.8 \\
\hline 13 & 160 & 1 & 13 & 153.5 & 97.4 & 94.9 to 99.5 \\
\hline 17 & 91 & 0 & 36 & 73.0 & 97.4 & 94.1 to 99.5 \\
\hline 18 & 55 & 0 & 20 & 45.0 & 97.4 & 93.3 to 99.5 \\
\hline 19 & 35 & 0 & 21 & 24.5 & 97.4 & 92.2 to 99.5 \\
\hline 20 & 14 & 0 & 11 & 8.5 & 97.4 & 89.4 to 99.5 \\
\hline 21 & 3 & 0 & 3 & 1.5 & 97.4 & 81.0 to 99.5 \\
\hline
\end{tabular}

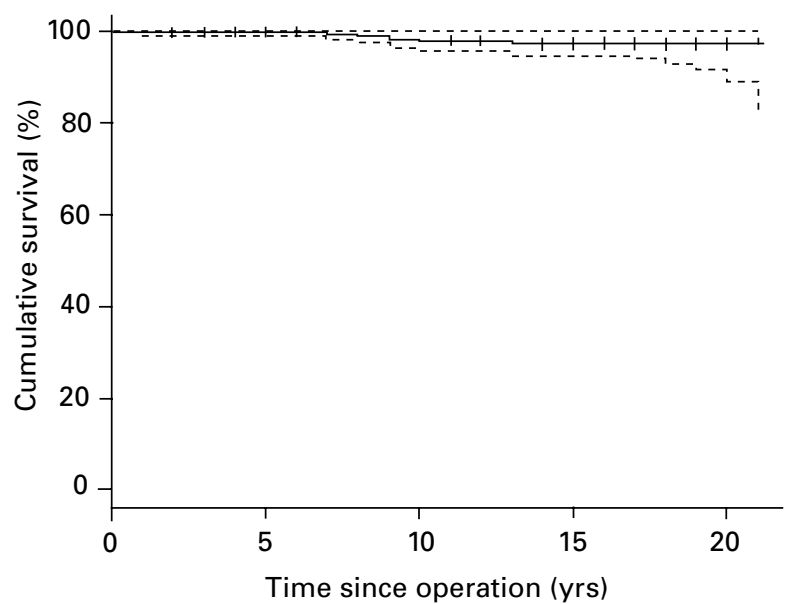

Fig. 3

Kaplan Meier survival curve (with 95\% confidence intervals) with revision of the femoral component for any reason as the endpoint.

There were 30 revisions $(9.1 \%)$ of the acetabular component for infection (3 hips), dislocation (4 hips), aseptic loosening (18 hips) and polyethylene wear (5 hips).

Per-operative fracture. There were 16 (4.8\%) per-operative fractures, all of which were iatrogenic fractures of the anterior femoral cortex. In 15 hips (15 patients) a minor per-operative proximal fracture of the femur was identified during implantation of the femoral component, as previously described. ${ }^{1}$ All patients with peroperative fractures identified during surgery were mobilised non-weight-bearing for six weeks. In one patient, a

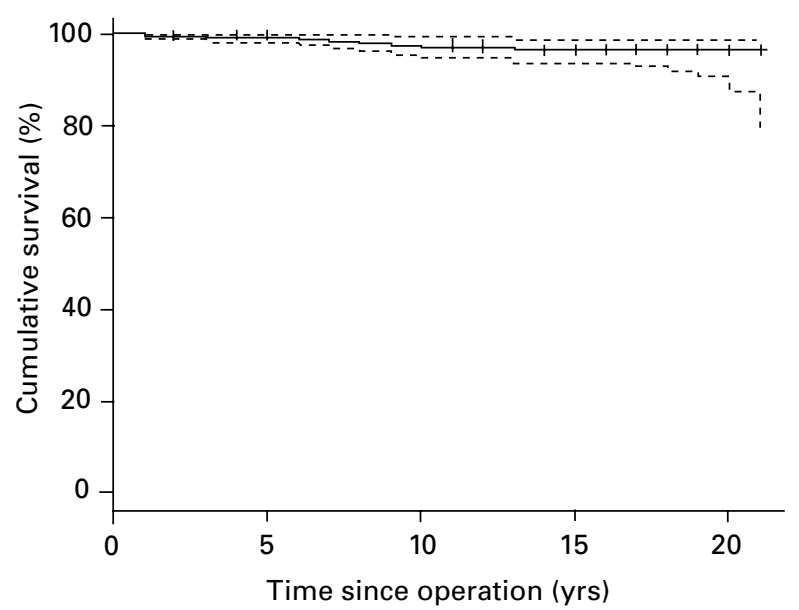

Fig. 4

Kaplan Meier survival curve (with 95\% confidence intervals) showing the worst case scenario. The two cases lost to follow-up have been included as stem failures.

cerclage wire was used to stabilise the fracture. All patients with iatrogenic proximal fractures of the femur sustained during insertion of the stem went on to unite satisfactorily, and did not suffer any long-term consequences.

Survival analysis. The life-table survival for the femoral component (including the six revisions) at a mean of 17 years was $97.4 \%$ (95\% confidence interval (CI) 94.1 to 99.5$)$ and at a maximum of 21 years was $97.4 \%(95 \%$ CI 81.0 to 99.5 ) (Table II, Fig. 3). Figure 4 shows the worst case scenario, including the two patients lost to follow-up. 


\section{Discussion}

Previous studies on the Furlong HA-coated femoral component have shown excellent medium-term survival when used as a primary ${ }^{1,2,5,6}$ or revision implant. ${ }^{6,7}$

Our current series includes the first 100 Furlong HAcoated THRs which were previously reported at a mean of ten years (9 to 12), with a $100 \%$ follow-up and a survival of $98.95 \%$ at up to 12 years, with no cases of aseptic loosening of the femoral stem. ${ }^{1}$

The Furlong HA-coated THR has also successfully been used in younger patients. ${ }^{5,6}$ Singh et $\mathrm{al}^{5}$ reported the successful use of this implant in patients below the age of 50 years with a $100 \%$ survival of the femoral component at a mean of ten years (5.3 to 14.2). Robertson et $\mathrm{al}^{6}$ had a $95.3 \%$ survival of the femoral component at a mean of 8.8 years (5 to 13.8) in patients less than 55 years of age. They did not identify any cases of aseptic loosening of the femoral component.

The Furlong HA-coated THR has provided impressive medium-term results as a revision implant. ${ }^{7,8}$ At a mean follow-up of eight years (5 to 12.4 ) Trikha et $\mathrm{al}^{7}$ reported survival of the femoral component of $100 \%$ with aseptic loosening as the endpoint, while Raman et $\mathrm{al}^{8}$ found similarly good results with survival of $95.6 \%$ at a mean follow-up of 12 years when revising cemented THRs.

In our series of 331 consecutive THRs, survival of the femoral component at a mean of 17 years was $97.4 \%$ (95\% CI 94.1 to 99.5$)$ and at a maximum of 21 years was $97.4 \%$ (95\% CI 81.0 to 99.5 ), with revision for any reason as the endpoint. With aseptic loosening of the femoral component as the endpoint, the survival would be $100 \%$. These figures are similar to those in the literature for the same femoral component. ${ }^{1,2,5-8}$

The length of follow-up achieved in this study is largely attributable to the static elderly population of our region. Only two of the 331 THRs were unaccounted for, thereby reducing the errors in the survival analysis highlighted by Murray, Britton and Bulstrode. ${ }^{9}$

The Merle D'Aubigne and Postel hip scoring system ${ }^{3}$ for the assessment of pain, mobility and function has been used since the commencement of this study. However, the mobility and function scores achieved at the latest follow-up were influenced by the advancing age of the patients as the follow-up extended. This explains the good mean scores of 5.63 for pain and 5.42 for mobility, but the relatively modest score of 4.50 for function.

The collar on the femoral component prevents early subsidence of the prosthesis after implantation. Once bone has bonded to the prosthesis, its function becomes redundant. This may account for the rounding of the bone under the femoral collar seen on the radiographs at the final follow-up.

Stress protection osteopenia occurs when a distally wellfixed femoral component takes the load in preference to the proximal femur. From the plain radiographs we were unable to identify any consistent pattern of osteopenia in our series and believe that the Furlong HA-coated femoral component loads the entire femur surrounding it adequately. However, we accept that we have not performed bone densitometry of the proximal femur to accurately quantify peri-prosthetic osteopenia.

A total of 16 peri-operative fractures occurred in our series, 11 of which were seen in the first 100 THRs, as reported previously, ${ }^{1}$ and comprised iatrogenic fractures of the anterior femoral cortex. The prevalence of these fractures in the early stages of the study most likely represents the learning experience encountered with the use of any new implant. Of the 16 fractures, 15 were proximal cracks of the anterior femoral cortex and did not require supplementary fixation. This type of fracture can be avoided by recognising that adequate space needs to be cleared in the posterolateral corner of the proximal femur to accommodate the relatively bulky body of the implant. One fracture extended further distally and required cerclage wire fixation to stabilise the implant. Nevertheless, all fractures united satisfactorily and there were no further complications.

Anterior thigh pain has been reported previously with the use of uncemented hip prostheses. ${ }^{10,11}$ This was not the case in our study. The absence of thigh pain in our study may be related to the modulus of elasticity of the titanium implant and to the bonding of bone throughout its length as shown in a retrieval analysis. ${ }^{12}$

We would like to thank our research assistants Mrs. K. Goddard, Ms K. Miles and Mrs. D. East for their invaluable efforts over the last 20 years in making this prospective study possible.

No benefits in any form have been received or will be received from a commercial party related directly or indirectly to the subject of this article.

\section{References}

1. McNally SA, Shepperd JAN, Mann CV, Walzac JP. The results at nine to twelve years of the use of a hydroxyapatite-coated femoral stem. J Bone Joint Surg [Br] 2000;82-B:378-82.

2. Shetty AA, Slack R, Tindall A, James KD, Rand C. Results of a hydroxyapatite coated (Furlong) total hip replacement: a 13-15 year follow-up. J Bone Joint Surg [Br] 2005;87-B:1050-4.

3. Merle d'Aubigne R, Postel M. Functional results of hip arthroplasty with acrylic prosthesis. J Bone Joint Surg [Am] 1954;36-A:451-75.

4. Gruen TA, McNiece GM, Amstutz HC. "Modes of failure" of cemented stem-type femoral components: a radiographic analysis of loosening. Clin Orthop 1979;141:1727

5. Singh S, Trikha SP, Edge AJ. Hydroxyapatite ceramic-coated femoral stems in young patients: a prospective 10 year study. J Bone Joint Surg [Br] 2004;86-B:111823.

6. Robertson A, Lavalette D, Morgan S, Angus PD. The hydroxyapatite coated JRIFurlong hip: outcome in patients under the age of 55 years. J Bone Joint Surg $[\mathrm{Br}]$ 2005;87-B:12-15.

7. Trikha SP, Singh S, Raynham OW, et al. Hydroxyapatite ceramic coated femoral stems in revision hip surgery. J Bone Joint Surg [Br] 2005;87-B:1055-60.

8. Raman R, Kamath RP, Parikh A, Angus PD. Revision of cemented hip arthroplasty using a hydroxyapatite-ceramic-coated femoral component. J Bone Joint Surg $[\mathrm{Br}]$ 2005;87-B:1061-7.

9. Murray DW, Britton AR, Bulstrode C. Loss to follow-up matters. J Bone Joint Surg [Br] 1997;79-B:254-6.

10. Engh CA, Bobyn JD, Glassman AH. Porous-coated hip replacement: the factors governing bone ingrowth, stress shielding and clinical results. J Bone Joint Surg [Br] 1987;69-B:45-55

11. Engh CA, Massin P. Cementless total hip arthroplasty using the anatomic medullary locking stem: results using a survivorship analysis. Clin Orthop 1989;249:141-56.

12. Furlong RJ, Osborn JF. Fixation of hip prosthesis by hydroxyapatite ceramic coatings. J Bone Joint Surg [Br] 1991;73-B:741-5. 